MATEC Web of Conferences 11,01044 (2014)

DOI: $10.1051 /$ matecconf / 20141101044

C) Owned by the authors, published by EDP Sciences, 2014

\title{
Méthode simplifiée de formulation d'un béton à haute performance avec et sans fibre métallique
}

\section{Simplified method design of high-performance concrete with and without steel fiber}

\author{
Hadj Sadok Ahmed ${ }^{1}$, Foudhil Cherif Imane ${ }^{2}$ et Si-Tayeb Said ${ }^{3}$ \\ ${ }^{1}$ Ecole Nationale Supérieure de l'Hydraulique, GEE, Blida, Algérie \\ ${ }^{2}$ Université de Saad Dahleb, Blida, Algérie \\ ${ }^{3}$ COSIDER, Alger, Algérie
}

\begin{abstract}
Résumé. Le présent travail, propose une méthodologie simplifiée, s'appuyant sur des méthodes de formulation de béton connues dans la littérature et sur des essais d'optimisations au laboratoire, permettant la détermination d'une composition d'un béton à haute performance "BHP » et de béton de fibre à haute performance «BFHP ». Pour ce faire, une large caractérisation (physique et mécanique) des matériaux utilisés a été conduite. Suivie d'une étude préliminaire, ayant pour objectif d'optimiser le dosage en Superplastifiant $(0,65-1 \%)$ et du ciment $(500-700) \mathrm{kg} / \mathrm{m}^{3}$ en se basant sur des essais de résistance à la compression $(3,7,28$ jours) et des mesures d'ouvrabilité. Les caractéristiques mécaniques ainsi que la porosité du BHP optimale ont été mesurées. Ainsi, l'effet d'une nouvelle génération de superplastifiant, qui a permis la réduction du rapport E/C à 0,29 , a pu être examiné. Ce dernier a permis l'obtention d'un BHP de bonne performance mécanique et de faible porosité. La méthodologie proposée a permis, à ce stade de l'étude, de formuler des bons BHP et reste à ajuster et à compléter pour la formulation des BFHP.
\end{abstract}

\begin{abstract}
This work proposes a simplified methodology, based on some literature methods of concrete formulation and tests of optimizations in laboratory, for the determination of a composition of a highperformance concrete "HPC" and high performance fiber concrete "HPFC". To do this, a broad characterization (physical and mechanical) of materials has been conducted. Followed by preliminary study aimed at optimizing the superplasticizer rate ( 0.65 to $1 \%$ ) and cement ( $500-700) \mathrm{kg} / \mathrm{m} 3 \mathrm{based}$ on compressive strength tests of $(3,7,28$ days) and workability measures. The mechanical characteristics and porosity of optimal HPC were measured. Thus, the effect of a new generation superplasticizer, which allowed the reduction of $\mathrm{W} / \mathrm{C}$ ratio at 0.29 , could be considered. The latter permits to obtain a HPC with a good mechanical performance and with low porosity. The proposed methodology allowed at this stage of the study, to make good HPC and remains to be adjusted and completed for HPFC design..
\end{abstract}

\section{Introduction}

Les applications modernes des constructions et d'ouvrages d'art, exigent de plus en plus des bétons performants tant sur le plan mécanique et de durabilité, que sur le plan de propriétés rhéologiques (ouvrabilité et homogénéité). Les bétons à hautes performances « BHP» et des bétons de fibre à haute performance " BFHP ", obtenus conjointement par l'emploi d'adjuvants et d'additions minérales présentent des résistances élevées et une longévité très appréciable $[1,2]$.
Les méthodes habituelles de formulation de béton, notamment celle de Dreux-Goriss, restent destinées aux bétons ordinaires qui, généralement, n'exigent que des dosages en ciment inférieurs à $450 \mathrm{~kg} / \mathrm{m} 3$, et des rapports E/C supérieurs à 0,4 toute en offrant un affaissement maximal de $12 \mathrm{~cm}$. Pour la formulation des BHP, d'autres méthodes ont été développées, entre autre, la méthode de Sherbrooke et la méthode de Baron-Lesage $[3,4]$ qui consistent globalement à fixer des paramètres de départ $(\mathrm{E} / \mathrm{C}$, ciment,...) et de cibler à travers plusieurs gâchées d'essai la maniabilité et la résistance souhaitées. 
Sur le marché actuel, de nombreux produits sont disponibles, notamment les superplastifiants de nouvelles générations, qui sont de plus en plus performants mais, présentent par fois des incompatibilités avec les liants utilisés, et leurs dosages restent très sensibles à la qualité des matériaux. Ceci rend par fois l'optimisation de la formulation du béton, par les méthodes su-citées, difficile et longue.

Le principe général de formulation des BHP, passe par la réduction de la porosité du béton. Cet effet est obtenu principalement en diminuant le rapport $\mathrm{E} / \mathrm{C}$ (en ajoutant un superplastifiant), et/ou en ajustant granulométrie par des additions ultrafines. Le travail présenté, rentre dans le cadre d'une étude en cours, qui tend à mettre en place une méthodologie simplifiée pour la formulation des bétons à hautes performances fibrés et non fibrés. La partie présentée dans cet article est une étude préliminaire qui examine principalement, l'optimisation des dosages de superplastifiant et du ciment. Les résultats de cette étude, permettrons ultérieurement de finaliser l'approche globale de formulation en traitant les volets additions ultrafines et adaptation de la méthode pour les BFHP.

\section{Caractérisation de matériaux et essais réalisés}

Le ciment utilisé pour la confection des bétons est un ciment Portland de type CEM II 42,5 N. Les compositions chimiques et minéralogiques du ciment sont données dans le tableau 1. A fin d'utiliser un sable « $\mathrm{S} »$ de finesse optimale, nous avons procédé par un mélange de deux (02) sables S1 (0/5) et S2 (0/1) avec les proportions respectives de $83 \%$ et $17 \%$. Aussi, deux (02) graviers, G1 (3/8) et G2 (8/15) ont été utilisés. Les caractéristiques physiques des granulats sont présentées dans le tableau 2. Le superplastifiant utilisé, commercialisé sous les noms « GLENIUM 51 » est un adjuvant non chloré de nouvelle génération à base d'éther polycarboxylique modifié. Ses caractéristiques sont présentées dans le tableau 3.

Tableau 1. Composition chimique du ciment.

\begin{tabular}{|c|c|c|c|c|c|c|c|c|}
\hline \multicolumn{9}{|c|}{ Compositions chimique minéralogique (\%) } \\
\hline $\mathrm{SiO}_{2}$ & $\mathrm{Al}_{2} \mathrm{O}_{3}$ & $\mathrm{Fe}_{2} \mathrm{O}_{3}$ & $\mathrm{CaO}$ & $\mathrm{SO}_{3}$ & $\mathrm{MgO}$ & $\mathrm{K}_{2} \mathrm{O}$ & $\mathrm{Na}_{2} \mathrm{O}$ & PAF \\
\hline 22.60 & 4.2 & 3.55 & 62.17 & 2.19 & 0.63 & 0.42 & - & 1.84 \\
\hline \multicolumn{2}{|c|}{$\mathrm{C}_{3} \mathrm{~S}$} & \multicolumn{2}{|c|}{$\mathrm{C}_{2} \mathrm{~S}$} & \multicolumn{3}{|c|}{$\mathrm{C}_{3} \mathrm{~A}$} & \multicolumn{2}{|c|}{$\mathrm{C}_{4} \mathrm{AF}$} \\
\hline \multicolumn{2}{|c|}{41.8} & \multicolumn{2}{|c|}{33.3} & \multicolumn{3}{|c|}{5.1} & \multicolumn{2}{|c|}{10.7} \\
\hline
\end{tabular}

Tableau 2. Caractéristiques physique des granulats d'études.

\begin{tabular}{|c|c|c|c|c|c|}
\hline Caractéristiques & $\begin{array}{c}\text { Sable } 0 / 5 \\
« \mathrm{~S} 1 »\end{array}$ & $\begin{array}{c}\text { Sable 0/1 } \\
\text { «S2 » }\end{array}$ & $\begin{array}{c}\text { Sable optimal* } \\
\text { «S } »\end{array}$ & $\begin{array}{c}\text { Gravier } \\
3 / 8 \\
\text { « } \mathrm{G} 1 »\end{array}$ & $\begin{array}{c}\text { Gravier } \\
8 / 15 \\
\text { «G2» }\end{array}$ \\
\hline Module de finesse & 3.3 & 0.94 & 2,9 & - & - \\
\hline Masse volumique apparente $\mathrm{g} / \mathrm{cm}$ & 1420 & 1380 & 1413 & 1435 & 1400 \\
\hline Masse volumique absolue $\mathrm{g} / \mathrm{cm}$ & 2690 & 2630 & 2675 & 2650 & 2650 \\
\hline Coefficient d'aplatissement « $\mathrm{A} »$ & - & - & - & - & 6.91 \\
\hline Equivalent de sable $\%$ & 84 & 79 & 82 & - & - \\
\hline
\end{tabular}

Tableau 3. Caractéristiques du superplastifiant

\begin{tabular}{|c|c|}
\hline Caractéristiques & Valeurs \\
\hline Masse volumique & $1,1 \pm 0,02 \mathrm{~g} / \mathrm{cm}^{2}$ \\
ph & $7,0 \pm 1$ \\
Teneur en chlorures & $<0,1 \%$ \\
Extrait sec & $35 \pm 2 \%$ \\
$\mathrm{Na}_{2} \mathrm{O}$ & $<2 \%$ \\
\hline
\end{tabular}

Dans l'étude de formulation du béton, l'ouvrabilité est mesurée par l'essai d'affaissement classique au cône d'Abrams selon la norme EN 12350-2. La résistance à la compression est mesurée sur des échantillons cubiques $(15 \times 15 \mathrm{~cm})$ confectionnés selon la norme EN 12390-2 et conditionnés dans l'eau à $20^{\circ} \mathrm{C}$. Des mesures de résistance de traction par flexion (sur éprouvettes prismatiques $7 \times 7 \times 28 \mathrm{~cm}$ ) ainsi que de porosité à l'eau (sur éprouvette cubique15x15cm) du BHP optimal ont été mesurées.

\section{Formulation des bétons}

Comme précisé, cette partie d'étude propose de formuler un BHP d'un rapport E/C efficace avec de bonnes caractéristiques rhéologiques, en passant par une optimisation des dosages de superplastifiant et du dosage en ciment. Pour cela, l'approche consiste à cibler une bonne ouvrabilité de départ (affaissement de18-25 cm). Après avoir déterminé le dosage en granulat, en fixant le 
rapport G/S et en adaptant la méthode de Dreux Goriss [5], l'étude passe par les deux étapes suivantes :

$\checkmark$ Détermination du dosage optimal en superplastifiant et du rapport $\mathrm{E} / \mathrm{C}$,

$\checkmark$ Détermination du dosage en ciment optimal.

La méthodologie de formulation est schématisée dans la figure 1.

\subsection{Détermination du dosage en granulats}

En fixant le rapport gravier sur sable $(\mathrm{G} / \mathrm{S}=1.1)$ et en estimant la compacité du béton par les coefficients de compacité donnés par G. Dreux [5], les compositions en granulats des bétons étudiés (B500, B600 et B700) sont présentées dans le tableau 4.

Tableau 4. Compositions en granulats des bétons étudiés $\left(\mathrm{kg} / \mathrm{m}^{3}\right)$

\begin{tabular}{|c|c|c|c|}
\hline Constituants & B 500 & B600 & B700 \\
\hline Ciment & 500 & 600 & 700 \\
Gravier 3/8 & 433 & 411 & 388 \\
Gravier 8/15 & 433 & 411 & 388 \\
Sable 0/1 & 134 & 126 & 118 \\
Sable 0/3 & 662 & 627 & 595 \\
\hline
\end{tabular}

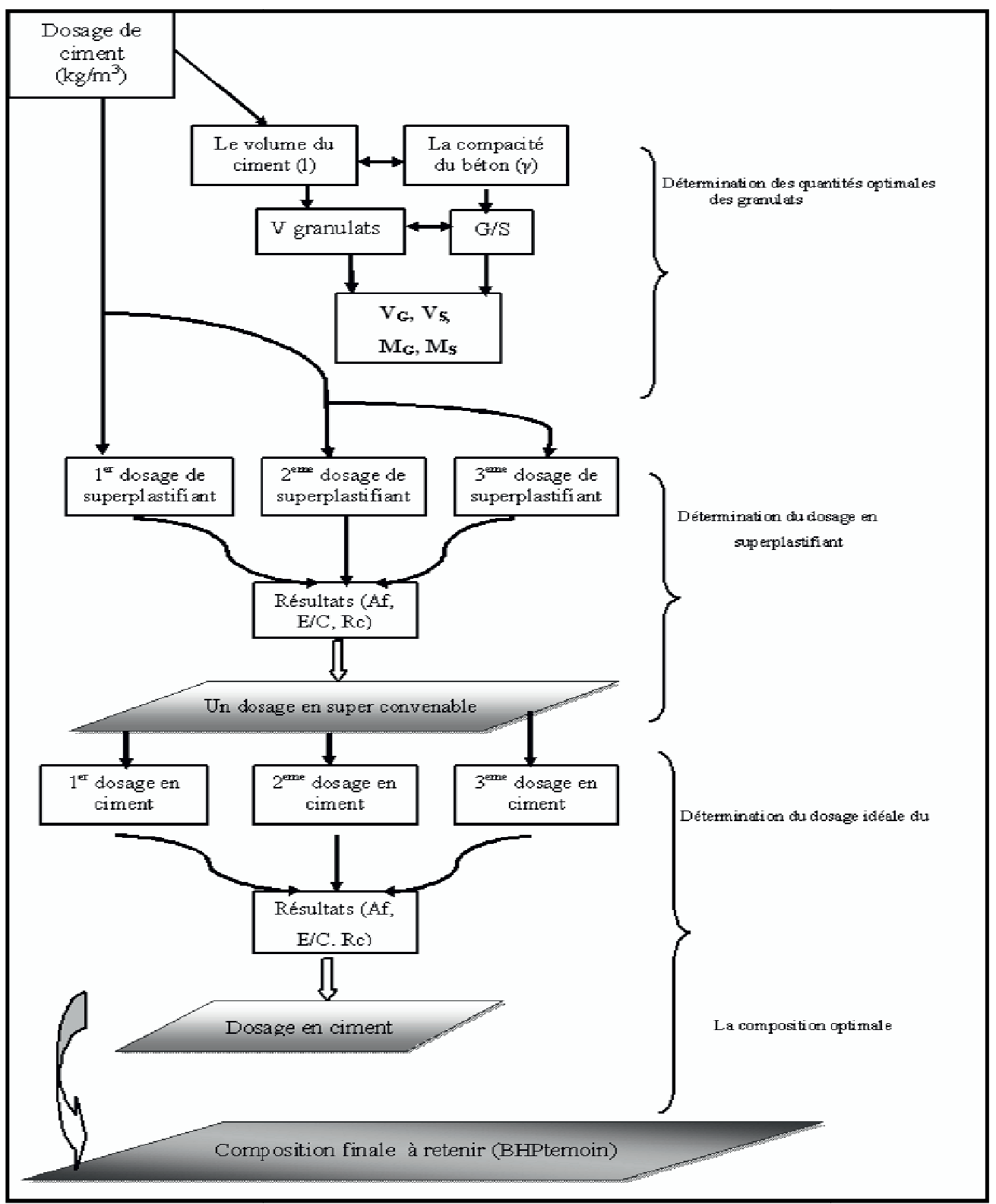

Fig.1. Méthodologie de formulation du BHP 


\subsection{Optimisation du dosage en superplastifiant et du rapport E/C}

Cette partie d'étude a été réalisée avec deux dosages en ciment (500 et $600 \mathrm{~kg} / \mathrm{m} 3)$. En ciblant la plage d'affaissement souhaitée, on varie le dosage en superplastifiant et on détermine le rapport $\mathrm{E} / \mathrm{C}$ correspondant. A fin d'avoir une idée primaire sur la qualité $\mathrm{du}$ béton à optimiser, la résistance à la compression des bétons étudiés a été mesurée à 7 et 28 jours. Les résultats sont regroupés dans le tableau 5 .

Les résultats montrent que le point de saturation en superplastifiant est au delà de $1 \%$. Néanmoins les dosages étudiés $(0.65,0.85$ et $1 \%)$ ont permis l'obtention d'un rapport $\mathrm{E} / \mathrm{C}$ assez faible $(<0.42)$ et de respecter l'ouvrabilité ciblée (affaissement $>20 \mathrm{~cm}$ ), notamment pour les bétons dosés à $600 \mathrm{~kg}$ de ciment où un rapport de $\mathrm{E} / \mathrm{C}$ de 0,29 a pu être obtenu. Ces résultats, nous ont permis de connaitre le dosage indicatif du superplastifiant pour un rapport E/C optimal. Ce dosage peut être ajusté pour d'autres dosages en ciment.

\subsection{Optimisation du dosage en ciment}

Afin d'ajuster le dosage en ciment, une étude de la résistance à la compression à 3,7 et 28 jours a été réalisée sur trois béton $\mathrm{B} 500, \mathrm{~B} 600$ et $\mathrm{B} 700$, ayant respectivement des dosages en ciment de 500,600 et 700 $\mathrm{kg} / \mathrm{m}^{3}$. Les quantités des granulats des bétons étudiés sont présentées dans le tableau 5. Pour ces bétons, le rapport $\mathrm{E} / \mathrm{C}$ de 0.29 a été maintenu fixe et le dosage de superplastifiant à $1 \%$. Les résultats obtenus sont présentés dans le tableau 6 et la figure 2 .

Tableau 5 : Effet de la variation du dosage en superplastifiant et du ciment sur le rapport $\mathrm{E} / \mathrm{C}$ et la résistance en compression.

\begin{tabular}{|c|c|c|c|c|c|}
\hline \multirow{2}{*}{$\begin{array}{l}\text { Dosage } \\
\text { en } \\
\text { Ciment }\end{array}$} & \multirow{2}{*}{$\begin{array}{c}\text { Dosage en } \\
\text { Super- } \\
\text { Plastifiant (\%) }\end{array}$} & \multirow{2}{*}{$\begin{array}{l}\text { Slump } \\
\text { A }(\mathrm{cm})\end{array}$} & \multirow[b]{2}{*}{$\mathrm{E} / \mathrm{C}$} & \multicolumn{2}{|c|}{$\mathrm{R}_{\mathrm{c}}(\mathrm{MPa})$} \\
\hline & & & & $\begin{array}{c}7 \\
\text { jours }\end{array}$ & $\begin{array}{c}28 \\
\text { jours }\end{array}$ \\
\hline \multirow{3}{*}{$\begin{array}{c}500 \\
\mathrm{~kg} / \mathrm{m}^{3}\end{array}$} & $0.65 \%$ & 20 & 0.40 & 49 & 56 \\
\hline & $0.85 \%$ & 23 & 0.42 & 47 & 55 \\
\hline & $1.00 \%$ & 21 & 0.34 & 54 & 60 \\
\hline \multirow{3}{*}{$\begin{array}{c}600 \\
\mathrm{~kg} / \mathrm{m}^{3}\end{array}$} & $0.65 \%$ & 23 & 0.36 & 52 & 50 \\
\hline & $0.85 \%$ & 20 & 0.31 & 51 & 61 \\
\hline & $1.00 \%$ & 24 & 0.29 & 52 & 82 \\
\hline
\end{tabular}

Tableau 6. Résistance en compression des bétons étudiés.

\begin{tabular}{|c|c|c|c|c|}
\hline \multirow{2}{*}{$\begin{array}{c}\text { Ciment } \\
\left(\mathrm{Kg} / \mathrm{m}^{3}\right)\end{array}$} & \multirow{2}{*}{$\mathrm{E} / \mathrm{C}$} & \multicolumn{3}{|c|}{ Resistance à la compression } \\
\cline { 3 - 5 } & & 3 jours & 7 jours & 28 jours \\
\hline 500 & 0.29 & 39 & 54 & 60 \\
\hline 600 & 0.29 & 54 & 55 & 80 \\
\hline 700 & 0.29 & 51 & 57 & 73 \\
\hline
\end{tabular}

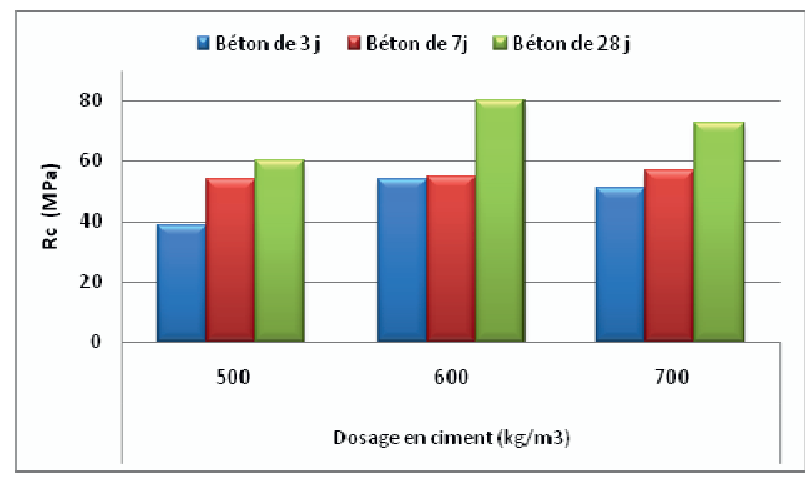

Fig. 2. Influence du dosage en ciment sur l'évolution de la résistance en compression.

\section{Caractérisation du BHP optimal}

Le béton $\mathrm{B} 600$ est considéré comme le BHP le plus performant, en se basant sur ses caractéristiques rhéologiques et mécaniques. Sa formulation ainsi que ses caractéristiques physiques et mécaniques sont présentées dans le tableau 7 et la figure 3. Ce dernier présente de bonnes caractéristiques mécaniques. En effet, on note une bonne évolution de la résistance à la compression (27 $\mathrm{MPa}$ à $24 \mathrm{~h}$ jusqu'à $82 \mathrm{MPa}$ à 28 jours). La porosité à l'eau mesurée à 28 jours, de $3.68 \%$, reflète une bonne compacité du béton étudié.

Tableau 7. Formulation et caractéristiques du BHP optimal.

\begin{tabular}{|l|c|}
\hline \multicolumn{2}{|c|}{ Formulation } \\
\hline Ciment & $600 \mathrm{~kg} / \mathrm{m}^{3}$ \\
\hline Gravier 3/8 & $433 \mathrm{~kg} / \mathrm{m}^{3}$ \\
\hline Gravier 8/15 & $433 \mathrm{~kg} / \mathrm{m}^{3}$ \\
\hline Sable 0/1 & $134 \mathrm{~kg} / \mathrm{m}^{3}$ \\
\hline Sable 0/5 Caractéristiques & $662 \mathrm{~kg} / \mathrm{m}^{3}$ \\
\hline Superplastifiant (1\%) & $5 \mathrm{~kg} / \mathrm{m}^{3}$ \\
\hline E/C & 0.29 \\
\hline & $19 \mathrm{~cm}$ \\
\hline Affaissement & 2.48 \\
\hline Densité & $82 \mathrm{MPa}$ \\
\hline Résistance à la compression à 28 jours & $3.68 \%$ \\
\hline Porosité à l'eau à 28 jours &
\end{tabular}




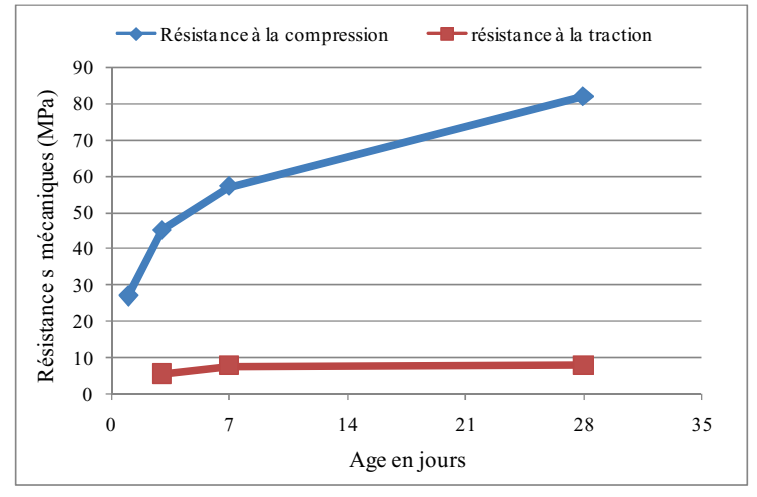

Fig. 3. Evolution des résistances mécaniques du BHP optimal

\section{Béton de fibre à haute performance « BFHP ॥}

A ce stade de l'étude, nous avons opté, pour la formulation du BFHP, à l'ajout d'une quantité de fibre métallique (Tableau 8 et figure 4) préconisée par la littérature [6] (en fonction des caractéristiques géométrique des fibres et du diamètre max des granulats) dans le BHP «B500» formulé antérieurement. Ainsi le dosage en fibre utilisé est de $20 \mathrm{~kg} / \mathrm{m}^{3}$ et les résultats obtenus du BFHP sont comparés avec ceux du BHP du même dosage en ciment $\left(500 \mathrm{~kg} / \mathrm{m}^{3}\right)$ dans le tableau 9 .

Le BFHP présente des caractéristiques moins performantes que celles du BHP du même dosage en ciment, d'un point de vu résistances mécaniques. Ceci reflète l'inconvénient d'introduire directement les fibres dans un béton ayant été préalablement optimisé sans fibres. En effet, cette introduction perturbe l'arrangement granulaire de la matrice, ce qui peut altérer la compacité du mélange [7]. Ceci montre l'importance d'optimiser la formulation d'un BFHP indépendamment du BHP. Cette approche fera l'objet d'une étude ultérieure.

Tableau 8. Caractéristique des fibres métallique.

\begin{tabular}{|c|c|c|c|c|}
\hline $\begin{array}{c}\text { Diamètre } \\
\mathrm{D}(\mathrm{mm})\end{array}$ & $\begin{array}{c}\text { Longueur } \\
\text { L }(\mathrm{mm})\end{array}$ & Densité & $\begin{array}{c}\text { Resistance } \\
\text { en traction } \\
(\mathrm{MPa})\end{array}$ & $\begin{array}{c}\text { Elasticité } \\
(\mathrm{MPa})\end{array}$ \\
\hline 0.75 & 60 & 7.8 & 1225 & 210000 \\
\hline
\end{tabular}

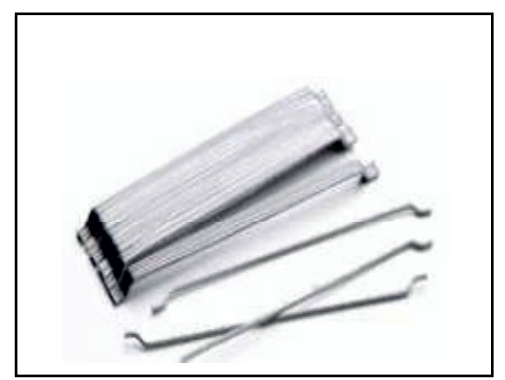

Fig.4. Fibres métalliques utilisés
Tableau 9. Les résultats résistances mécaniques du BHP et BFHP.

\begin{tabular}{|c|c|c|c|c|c|}
\hline Béton & \multicolumn{3}{|c|}{ BFHP } & \multicolumn{2}{c|}{ BHP } \\
\hline E/C & \multicolumn{3}{|c|}{0.32} & \multicolumn{2}{c|}{0.32} \\
\hline Ouvrabilité & \multicolumn{3}{|c|}{$22 \mathrm{~cm}$} \\
\hline $\begin{array}{c}\text { Résistances } \\
\text { mécaniques }\end{array}$ & $\begin{array}{c}3 \\
\text { jours }\end{array}$ & $\begin{array}{c}7 \\
\text { jours }\end{array}$ & $\begin{array}{c}28 \\
\text { jours }\end{array}$ & $\begin{array}{c}7 \\
\text { jours }\end{array}$ & $\begin{array}{c}28 \\
\text { jours }\end{array}$ \\
\hline $\begin{array}{c}\text { Compression } \\
(\mathrm{MPa})\end{array}$ & 29 & 45 & 53 & 54 & 60 \\
\hline $\begin{array}{c}\text { Traction par } \\
\text { flexion (MPa) }\end{array}$ & 5.00 & 6.62 & 6.8 & \multicolumn{3}{|c}{-} \\
\hline
\end{tabular}

\section{Conclusion}

La méthodologie proposée, même si incomplète à ce stade de l'étude, reste simple et permet l'optimisation d'un BHP de base avec de bonnes performances rhéologiques et mécaniques. Les résultats de cette étude serviront comme base à l'étude globale qui s'intéressera à l'optimisation de la substitution du ciment par des additions ultrafines ce qui permettra l'obtention de BHP de meilleures performances, notamment sur le plan de durabilité. Une adaptation de la méthodologie pour la formulation des BFHP indépendamment de la formulation des BHP reste indispensable.

\section{References}

1. R . Lakhal. D . Achoura, Elaboration des bétons à haute performances à base de laitier granulé (1st International Conference on sustainable Built Environment Infrastructures in Developing Countries ENSET Oran, Algérie, 2009).

2. E. Vejmelkov et al, High performance concrete containing lower slag amount: A complex (Const and Buil Mat. 2237-2245 (2009).

3. A. Pierre Claude, Bétons Haute Performance (Eyrolles 2001).

4. P. ROSSI, Le développement industriel des bétons de fibres métallique (ENPC, Paris, 2002)

5. J. Festa \& G. Dreux, Nouveau Guide du Béton et de ses Constituants (Eyrolles, 8 ième édition 1998).

6. Guide de bonnes pratiques pour l'utilisation des fibres dans le béton, (ABQ 2005).

7. P. ROSSI, les bétons de fibres métalliques, (ENPC, Paris, 1998). 